\title{
Basic Symptoms of Cluster Headaches
}

\section{Conceptual Paper}

The symptoms of cluster headaches appear abruptly and the pain is incredibly severe. Many times described by completely terrified patients getting their first attack as thinking they are having an aneurysm or stroke, a ruptured blood vessel bleeding out in the brain, etc. Sometimes the feeling of being shot in the head and with most all cases patients consistently describe the horrific real and terrifying feeling that death is most eminent and only moments away caused by such powerful and violent pain. It can go from no pain to peaking with powerful intensity in just minutes and can last from fifteen minutes to three hours multiple times daily and nightly but rarely longer than three hours per attack. The pain is caused by irritation \& activation of the branches of the trigeminal nerve (see illustration below).

We do not fully understand what causes cluster headaches and there are several theories out there. Considered at this point an incurable deep brain neurological pain syndrome, however research has been seriously lacking throughout it's history and with more research will ultimately give us a much better understanding of the condition. The hypothalamus and activation of it's surface gray matter is part of this and $\mathrm{CH}$ is a circadian disorder and the circadian body clock is the hypothalamus also involving the sphenopalatine ganglion. $\mathrm{CH}$ is repetitive and when attacks come normally if episodic $\mathrm{CH}$ vs being triggered, attacks can happen at the exact same time every day, night or day, however night time attacks seem to be more common with the disorder.

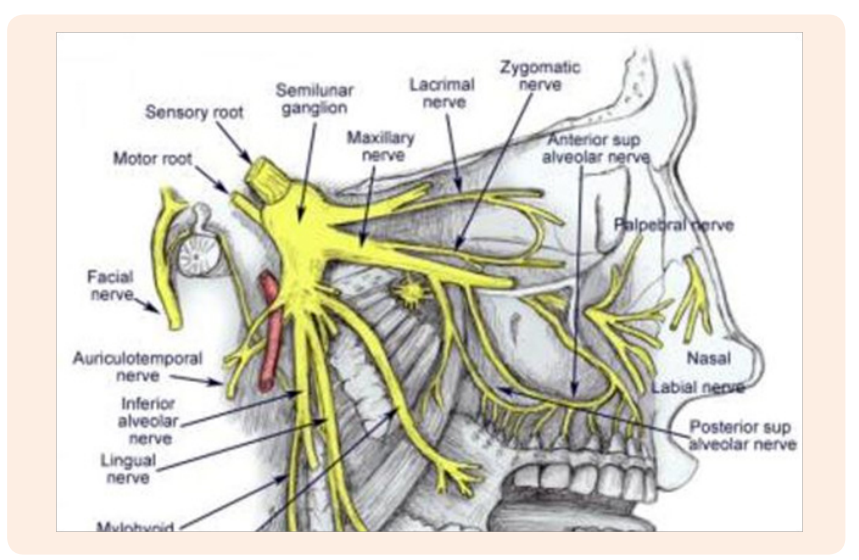

Attack cycles starting at the same time every year or every few years also possibly more than one attack cycle per year is not uncommon with varying cycle lengths lasting commonly weeks to months. The chronic form of the disorder with 2 to 8 or more attacks daily and or nightly lasting a full year with no more than 30 days of relief is the precise definition.

The pain is located on one side of the head behind, in or around one of the eyes effecting the temple and head on that same side as

\author{
Conceptual Paper \\ Special Issue - 2016 \\ John Fletcher* \\ President/Founder, Cluster Headache Foundation Inc., USA \\ *Corresponding author: John Fletcher, President/ \\ Founder Cluster Headache Foundation Inc., USA, Email: \\ clusterheadaches@yahoo.com \\ Received: December 18, 2015 | Published: April 06, 2016
}

well as severe neck pain and stiffness in the shoulders also painful. The intense violent stabbing pain will last between 15 minutes to 3 hours and may be accompanied by redness of the eye with excessive tearing, nasal congestion or runny nose, swelling of the eyelid, forehead or facial sweating, severe restlessness, agitation and some case sensitivity to light.

Analogies of this extremely severe pain have been described as feeling like a hot poker being inserted into the eye, a knife being pounded in and out of the eye and temple, an ice pick being shoved into the eye into the brain, etc.

Severe anxiety and heart palpitations are not uncommon as the attack begins and during an attack. Pain and other symptoms occur on the same side of the head. These attacks can be numerous and daily averaging 2 to 8 attacks daily is common and sometimes over a dozen or more day and night depending on the patient. They have the ability to wake the patient from a sound sleep with the most severe pain peaking in seconds to minutes with powerful intensity.

The disorder seems to be greatly affected by the seasons of the year and are affected by weather, altitude, a falling barometer, fall $\&$ winter seasons seem to be more of a peak time of year and many sufferers struggle through these seasons getting more frequent and significantly more powerful attacks. However, everyone is different in many ways and this is definitely not a one size fits all disorder. Summer heat is a known trigger, an indoor heater or central heating in the home seems to also be a trigger. Strong smells like perfumes or chemical smells, bright light, alcohol, especially red wines, fermented food and drink products also nitrites in processed meats... These can all be strong triggers for cluster attacks.

$\mathrm{CH}$ is not something that only happens once or twice or even several times, but a life long disorder from when it starts for most which can basically be at any age. The average age of onset is between twenty and thirty years old, but can start as young as two or three years old and is thought to be possible in even younger infants. Some cases of the crying baby syndrome are migraine or possibly $\mathrm{CH}$ or other headache disorders. Having a good neurologist/specialist to help and getting a solid diagnoses 
is very important, however $\mathrm{CH}$ is a complicated disorder and can be difficult to diagnose in many cases although getting a correct diagnoses is much better these days. Getting a diagnoses for $\mathrm{CH}$ used to take sometimes 10 to 20 years and not uncommon being considered a rare disease at 0.1 to $0.3 \%$ of the population.

In the last 20 years, diagnoses have improved dramatically, however still can take several years for a correct diagnoses. A study on the patients part and use of a headache log can improve chances of a correct diagnoses dramatically and is highly recommended if you are not diagnosed or incorrectly diagnosed.

REM sleep is also thought to possibly be a trigger for attacks, however, some sleep studies suggest that may not be the case. As the patient tries to sleep getting an attack within 15 to 20 minutes is not uncommon, but within one to two hours is very common bringing in another factor of a sleep disorder and severe sleep deprivation is commonly the result being one of several different problems that can be created by cluster headaches.

PTSD is a factor for many patients caused by such severe and traumatic attacks that some are never forgotten and flash backs of those devastating attacks can bring PTSD into play. Also clinical depression is common with this disease. These are also factors that can be treated by relieving the disease itself or treating them separately as a temporary means of symptom relief, but the disorder is the root cause of these powerful symptoms and treating some symptoms like depression without relieving the cause is not always successful... For more detailed information on this site See "An education about cluster headaches", "Understanding this disorder" or "Why Cluster Headaches Are Called Suicide Headaches". A new published description for more detail on the disorder is A General Description Of Cluster Headache Disorder

The following is a description of the violent pain caused by a severe cluster attack written by a long time $\mathrm{CH}$ patient... Considered by medical professionals worldwide as The most severe pain a human can experience...

\section{The True Essence of Cluster Headache Pain}

A Cluster Headache is just pure unadulterated crisp pain. Awesome in it's crystal clarity, pain far beyond the ultimate severity, pain beyond belief, pain beyond the realm of comprehension!

Just incredibly Violent Pain! Pain in it's purest, rawest clinical form. A Cluster is devastatingly brutal in it's existence and severely persistent in it's relentless assault. Penetrating deep into your soul. Searching for your weakness, many times finding it...

A Cluster completely violates your being and conquers the mind, ripping \& tearing through the eye, into your mind, body and soul, demanding your total respect and complete undivided attention, awareness and focus...

A Cluster completely obliterates your thoughts and your whole being. The pain consumes all of you overtaking all thought, destroying the ability to fight back... Absorbing you and violently dragging you into a deep dark abyss of pure primal all consuming terrifying pain and severe brutal agony. A void hell of existence where only excruciating pain thrives, abounds and exists...

Down there your only defense is raw primal instinct of self preservation and pure thought of survival above all else. A lonely dark excruciatingly painful and terrifying hell, always on the edge of the abyss, always on the edge of one last breath. Pushing us to the utmost limits of endurance and extreme stubborn resistance...

Pain so crisp and untainted and overwhelmingly intense there is nothing to compare it to... It has to be felt to be believed and even then is just unbelievable!... And although you can feel that you are most definitely without a doubt going to die, it doesn't kill you!! It leaves you embedded as a prisoner to come back and violate you and torture you every day and night, over and over and over again....

And in a sick, cynical cycle, we somehow survive it over and over and over again. A cruel \& terrifying ritual beyond any logic, far beyond the realm of imagination, far beyond conceived reality. It is the literal feeling of death without dying... Sometimes lasting for more than 40 years. In my case 41 years"...

-Hennie Koch, 2015 